\title{
Illustrations of Mathematical Modeling in Biology: Epigenetics, Meiosis, and an Outlook
}

\author{
D. RICHARdS, ${ }^{1,3}$ S. BerRY, ${ }^{2,3}$ AND M. HowARD ${ }^{2}$ \\ ${ }^{1}$ Division of Molecular Biosciences, Imperial College London, SW7 2AZ, United Kingdom \\ ${ }^{2}$ Department of Computational and Systems Biology, John Innes Centre, Norwich Research Park, \\ Norwich NR4 7UH, United Kingdom \\ Correspondence: martin.howard@jic.ac.uk
}

\begin{abstract}
In the past few years, mathematical modeling approaches in biology have begun to fulfill their promise by assisting in the dissection of complex biological systems. Here, we review two recent examples of predictive mathematical modeling in plant biology. The first involves the quantitative epigenetic silencing of the floral repressor gene FLC in Arabidopsis, mediated by a Polycomb-based system. The second involves the spatiotemporal dynamics of telomere bouquet formation in wheat-rye meiosis. Although both the biology and the modeling framework of the two systems are different, both exemplify how mathematical modeling can help to accelerate discovery of the underlying mechanisms in complex biological systems. In both cases, the models that developed were relatively minimal, including only essential features, but both nevertheless yielded fundamental insights. We also briefly review the current state of mathematical modeling in biology, difficulties inherent in its application, and its potential future development.
\end{abstract}

During the last decade, mathematical modeling has finally established itself as a mainstream methodology in the analysis of biological systems. The daunting complexity of biology, with the systems-level emergence of behaviors not present at the level of individual molecules, decidedly favors a modeling approach. In this chapter, we examine two applications of mathematical modeling in two very different areas of biology: one in epigenetic regulation and the other in the spatiotemporal dynamics of meiosis. In both cases, the specific application is to plants (Arabidopsis and wheat-rye), but the overall conclusions are likely to be widely applicable.

Despite their considerable differences, both systems show one overall similarity, namely, their ability to reliably function even in the presence of high levels of fluctuations. For the case of epigenetics, genes can be stably silenced despite the large fluctuations introduced, for example, at DNA replication. For the case of meiotic dynamics, the telomere bouquet reliably forms on a relatively short timescale, despite, for example, the potentially disordered nature of the cytoskeleton. In this review, we highlight this unifying issue and emphasize the minimal nature of the modeling. In biology, it is all too easy to use a mathematical model that is overly complex and therefore underconstrained by the available data. Rather, our approach has been to construct minimal models that can nevertheless make unexpected, ideally relatively qualitative, predictions about the focused system in question. These predictions can then be experimentally tested and the results fed back into further model refinement.

In our conclusion, using the above two studies as examples, we briefly comment on the future of mathematical modeling in biology, discussing in particular its relationship with bioinformatics. Our hope is that the potential of modeling will become even more widely exploited in the years ahead so that the complex problems of biology, in plants and beyond, can be dissected more efficiently.

\section{EXAMPLE 1: CHROMATIN-BASED EPIGENETICS}

Epigenetic change is often defined as a change in the expression state of a gene not involving a mutation but that is nevertheless inherited even in the absence of the initiating signal. Epigenetic memory has been studied for many years in systems such as the lysis-lysogeny switch in bacteriophage $\lambda$ and the lac operon in Escherichia coli. In both of these systems, the epigenetic information is stored globally, in the concentration of a diffusible factor. Positive feedback loops stabilize this concentration so that the state is stable and heritable through cell division (Ptashne and Gann 2002). It has been proposed more recently that regions of chromatin can be programmed into repressive and active states that control the expression states of their underlying genes and are mitotically heritable (Moazed 2011). In this case, epigenetic memory is thought to be stored locally in the structure of the chromatin. To store heritable information, this chromatin "state" must, however, be self-perpetuating despite noisy nucleosome removal, DNA replication and mitosis. Exactly how this is accomplished is currently the subject of much research.

An example of epigenetic memory in which information is believed to be encoded locally is in gene regulation by the Polycomb and trithorax groups $(\mathrm{PcG} / \mathrm{trxG})$ of

\footnotetext{
${ }^{3}$ These authors contributed equally to this work.

Copyright (C) 2012 Cold Spring Harbor Laboratory Press; all rights reserved; doi: 10.1101/sqb.2013.77.015941

Cold Spring Harbor Symposia on Quantitative Biology, Volume LXXVII
} 
proteins. These proteins were initially discovered in Drosophila, in which mutants exhibit homeotic transformations caused by misexpression of key developmental regulatory genes. The expression state of $\mathrm{PcG} / \mathrm{trxG}$ targets is inherited by daughter cells and $\mathrm{PcG} / \operatorname{trxG}$ components are often continuously required for maintenance of gene expression throughout development (Schwartz and Pirrotta 2007). Polycomb complexes have also been characterized in other multicellular eukaryotes including Arabidopsis thaliana, in which they are also required for epigenetic repression of developmental genes (Hennig and Derkacheva 2009).

\section{QUANTITATIVE EPIGENETIC REPRESSION OF FLC}

In Arabidopsis, prolonged exposure to cold temperatures accelerates flowering in a process called vernalization. Prolonged cold causes epigenetic repression of FLOWERING LOCUS C (FLC), a gene that encodes a MADS domain protein that negatively regulates the expression of genes that promote the floral transition. This process allows winter-annual Arabidopsis accessions to repress flowering until after a period of prolonged cold, to align flowering and seed production with spring (Crevillen and Dean 2010). FLC repression is epigenetic in the sense that it remains stable on return to warm conditions and quantitative in the sense that longer periods of cold exposure lead to progressively lower levels of FLC expression (Fig. 1A).

A necessary component of Polycomb-mediated gene repression is thought to be covalent modifications to histones at Polycomb target genes. Specifically, Polycomb repressive complex 2 (PRC2) is responsible for histone H3 lysine 27 trimethylation (H3K27me3). Before cold exposure, a core PRC2 complex is present at FLC (Fig. 1B). However, after cold exposure, additional plant homeodomain (PHD) proteins are found to be associated with the core PRC2 (Fig. 1C) (De Lucia et al. 2008). Recruitment of PHD proteins during the cold coincides with the deposition of H3K27me3 immediately downstream from the transcription start site of FLC, at the so-called "nucleation region" (Fig. 1C), possibly targeted by a specific DNA sequence. Subsequently, on return to the warm, both H3K27me3 and PHD-PRC2 complexes spread over the gene body leading to stable epigenetic repression of FLC (Fig. 1D) (De Lucia et al. 2008).

Although H3K27me3 over the gene body appears to be necessary for epigenetic FLC repression, genetic studies suggest that it is not sufficient. For example, plants containing mutations in VERNALIZATION 1 (VRN1) show increases in $\mathrm{H} 3 \mathrm{~K} 27 \mathrm{me} 3$ levels in response to

A
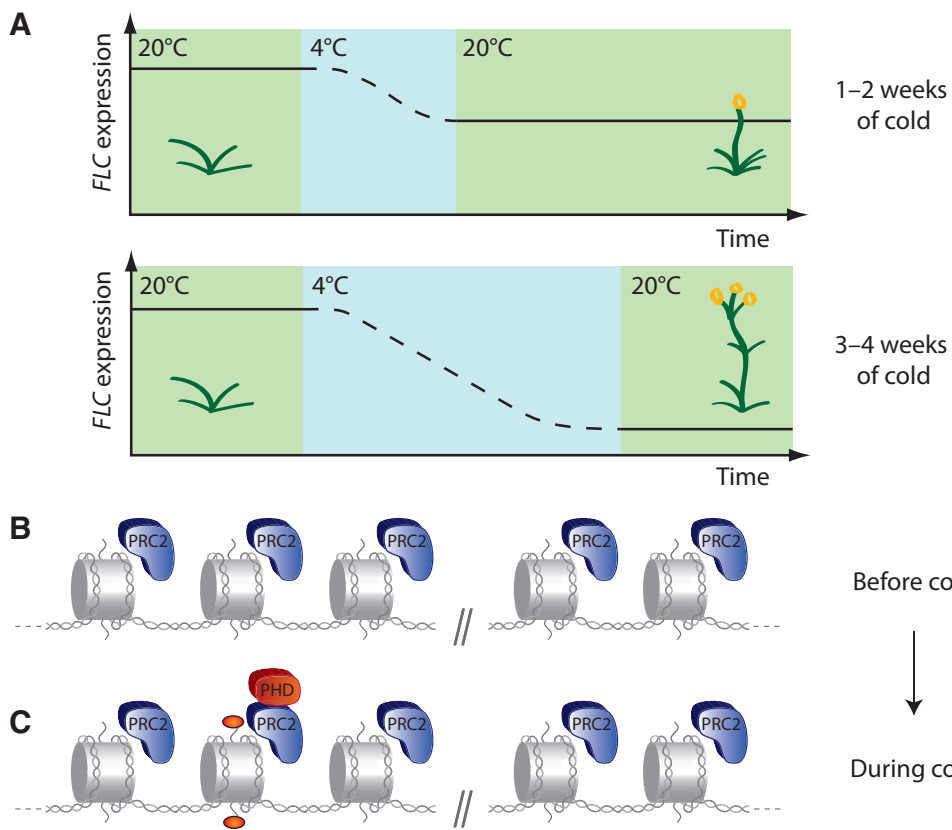

Before cold

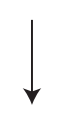

During cold
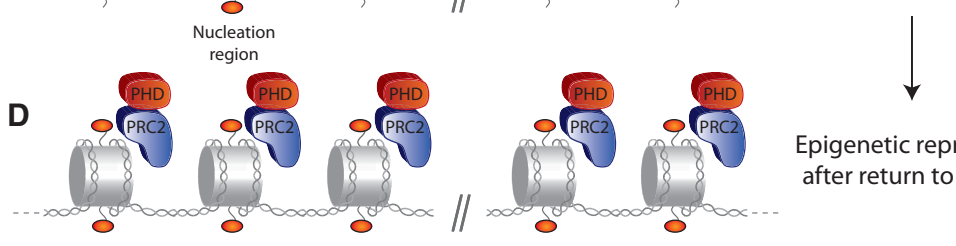

Epigenetic repression after return to warm

Figure 1. FLC expression and chromatin state during vernalization. (A) FLC is stably repressed in response to cold exposure. Longer periods of cold result in greater $F L C$ repression and earlier flowering after the return to warm. $(B) F L C$ chromatin during vernalization. Core PRC2 is present on FLC chromatin before cold exposure. (C) After cold exposure, H3K27me3 (red ovals) accumulates together with PHD-PRC2 complexes at the nucleation region. $(D)$ After a transfer back to warmer temperatures, H3K27me3 and PHD-PRC2 complexes spread outward from the nucleation region to cover the FLC gene body. (Adapted from Song et al. 2012.) 
vernalization, yet they fail to maintain repression of FLC during the subsequent warm (Bastow et al. 2004).

Several components of the PHD-PRC2 complex responsible for vernalization are conserved in other higher eukaryotes and it has been shown that the complex contains modules for both deposition of H3K27me3 and binding H3K27me3. These results have led to the proposal that PRC2 acts to "read" and "write" H3K27me3, to maintain a region of chromatin in a repressed state (Hansen et al. 2008; Margueron et al. 2009). This positive feedback is a central idea in the mathematical model of cold-induced $F L C$ repression proposed by Angel et al. (2011).

\section{MATHEMATICAL MODEL OF VERNALIZATION}

Although many of the components implicated in epigenetic silencing of FLC had been identified, as described above, a mechanistic understanding of how the pieces fit together into a coherent whole had not been formulated. In particular, it was unclear how this complex system epigenetically stores quantitative information during the duration of cold exposure. Consequently, we turned to mathematical modeling (Angel et al. 2011) to help to resolve these issues. We used an underlying framework from earlier theoretical work on stochastic switching of the epigenetic state at the Schizosaccharomyces pombe mating-type locus (Dodd et al. 2007). In the minimal model of Angel et al. (2011), histones can either be unmarked or in one of two opposing modification "states." Each of these opposing states recruits protein complexes that deposit more of their respective modification and remove the opposing modification from other histones in the locus (Fig. 2A). Under the continual action of such a mechanism, the histone modification status of a region of chromatin can become self-sustaining: Histone modifications, which are lost by nucleosome swap-out, noisy enzymatic activity, or DNA replication, will be replaced by protein complexes that deposit new modifications of the same kind. In part, the stability of these chromatin domains comes from their size, with larger regions being inherently more stable. However, two other conditions have also been shown to be required for stability: cooperativity in the action of the histone-modifying enzymes (see Fig. 2A) and long-range interactions between histones in the locus. This minimal model is clearly a gross oversimplification of the actual in vivo dynamics, yet, as we will see below, it is able to make testable predictions.

Dodd et al. (2007) showed that systems with mutually antagonistic histone modifications can lead to bistable regions of chromatin, whereas Angel et al. (2011) showed how switching between these active and repressed states could be controlled to quantitatively modulate the expression of a gene in response to an environmental stimulus. At the single-cell level, models of this type have only two stable states. However, the quantitative nature of FLC repression in response to cold implies a continuum

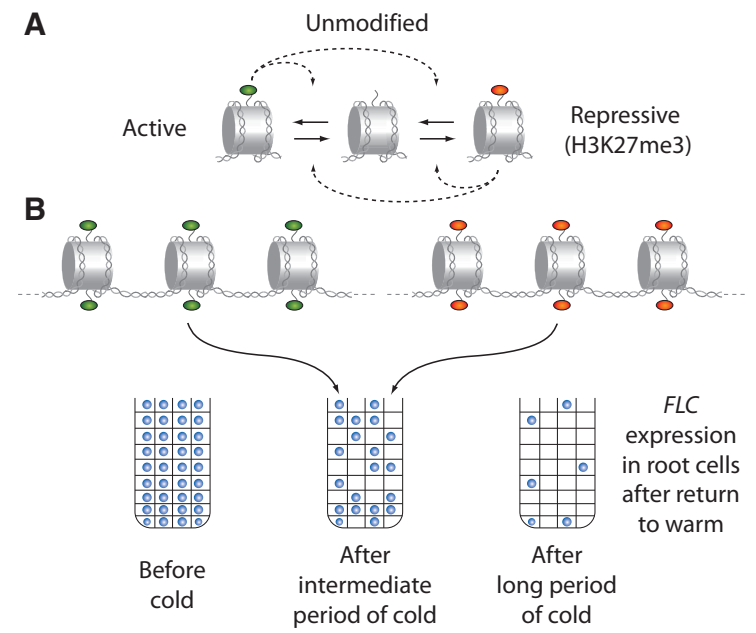

Figure 2. Key modeling principles and the quantitative nature of the vernalization response. (A) Cooperative positive feedback. Active histone modifications (green ovals) encourage further active modifications to be added and repressive modifications (red ovals) to be removed. Similarly, repressive modifications encourage further repressive modifications and the removal of active modifications. A requirement for two-step transitions, that is first passing through the unmodified state, ensures nonlinear cooperativity. $(B)$ The quantitative nature of the vernalization response is the result of an increasing number of cells switching to a repressed state after an increasing period of cold exposure. $F L C$ expression in individual cells can be observed directly in roots using an FLC: GUS reporter gene. In blue cells, $F L C$ is expressed, whereas in white cells, FLC is repressed. (Adapted from Song et al. 2012.)

of stable states at the whole-organism level. In the vernalization model, after cold exposure, each cell can be in either a high FLC expression state (with low H3K27me3 over the gene) or a low FLC expression state (with high H3K27me3 over the gene). As in the model of Dodd et al. (2007), epigenetic stability is generated by histone modification dynamics with nonlinear positive feedback. However, in the vernalization model, increasing duration of cold exposure leads to a greater proportion of cells switching from a low to a high $\mathrm{H} 3 \mathrm{~K} 27 \mathrm{me} 3$ state that generates a quantitative change in $F L C$ expression (Fig. 2B). This switching, which occurs when plants are returned to the warm, is enabled by quantitative cold-induced accumulation of $\mathrm{H} 3 \mathrm{~K} 27 \mathrm{me} 3$ in the nucleation region of $F L C$ that is then able to use the noisy histone modification dynamics to flip the epigenetic state of the whole locus to one predominantly covered by H3K27me3 (see Fig. 1C,D). The prediction of cell-autonomous repression was a key output of mathematical modeling. This prediction was subsequently verified experimentally using an FLC:GUS reporter to monitor FLC expression in individual cells of Arabidopsis roots (Fig. 2B) (Angel et al. 2011), revealing a digital on/off pattern of gene expression.

The digital nature of this histone-modification-based memory allows the expression of $F L C$ to remain stable through many rounds of DNA replication because each cell only has to sustain one of two possible epigenetic states. The modeling and experimental work described in 
Angel et al. (2011) shows that such a system can generate quantitative changes in gene expression at the whole-organism level despite having only two stable expression states in individual cells. If instead, however, each cell were to individually remember a graded level of FLC expression, the system would need a continuum of stable states within individual cells. Such a graded memory could potentially be much more difficult to sustain because of disruption by fluctuations.

In the model of Angel et al. (2011), the memory of cold exposure is directly encoded in the chromatin of FLC. There is currently much discussion in the literature about whether histone modifications are a fundamental memory element capable of recruiting the machinery necessary for the maintenance and heritability of epigenetic states (Kaufman and Rando 2010). The vernalization model does not provide the answer to this question but instead analyzes the mechanistic features that a histone-modification-based memory system requires to be capable of generating stable alternative expression states that can be switched in response to an environmental stimulus. Interestingly, it has recently been suggested that Polycomb-mediated epigenetic memory through DNA replication may be mediated through persistent binding of $\mathrm{PcG} / \operatorname{trxG}$ proteins to the locus in question rather than by the histone modifications themselves (Petruk et al. 2012). In this context, we note that a memory system that relies on such enzyme binding would actually be very similar in structure to the model of Angel et al. (2011), with the activating/silencing histone modifications simply relabeled as the presence/absence of activating/silencing proteins. Determining the actual nature of the memory element(s), which could vary from one system to another, is clearly a key aim for future experiments.

\section{EXAMPLE 2: MEIOTIC TELOMERE DYNAMICS}

Our second modeling example is concerned with the spatiotemporal dynamics of meiosis. How genetic material is passed on to the next generation is obviously a crucial step in the life cycle of all organisms. This process was revolutionized by the evolution of meiosis, in which the number of chromosomes is halved in cells destined to become gametes. However, meiosis necessarily introduces many complications, not the least of which is the necessity of pairing related (homologous) chromosomes to ensure that each gamete receives the full haploid genome. Homologous pairing, about which relatively little is known, poses many intriguing questions such as how chromosomes search for their partners and how homologous chromosomes are able to recognize one another while avoiding nonhomologous associations.

At first glance, the homologous chromosome search seems to be an almost impossible task, whereby whole chromosomes (each vastly larger than the size of a typical protein) must navigate the entire nucleus in search of their single unique partner (Moore and Shaw 2009).
Organisms tend to mitigate this problem by attaching the telomeres to the nuclear membrane during meiosis. With telomeres confined to the membrane, subtelomeric regions have a much smaller region to search for their homologous partners. In fact, many organisms go further and move all of the telomeres to a small region on the nuclear membrane, forming a chromosome configuration called the telomere bouquet that potentially even further reduces the search space (Zickler and Kleckner 1998; Scherthan 2001, 2007; Harper et al. 2004).

\section{FORMATION OF THE TELOMERE BOUQUET}

How the telomere bouquet is formed has long been mysterious, especially because the time for bouquet formation is similar in many organisms, even those as diverse as yeast and wheat. This is especially surprising because wheat is a "worst-case-scenario" organism for meiosis with more than 1000 times more DNA (17 Gb) and more than eight times the nuclear radius $(8 \mu \mathrm{m})$ compared with yeast. Furthermore, bread wheat is hexaploid, with three related, although nonhomologous, sets of chromosomes. Hence, identification of the correct homologous pairs is potentially even more difficult owing to the necessity of preventing incorrect pairings among related but nonhomologous chromosomes. In this context, the Phl locus, a region on chromosome 5B that has been localized to a cluster of defective Cdk-like genes (Greer et al. 2012), is of vital importance. It seems to have evolved, at least in part, to help avoid these incorrect nonhomologous pairs. Its mechanism of action is not yet understood, although the initiation of bouquet formation seems to occur earlier in a mutant without Phl (Richards et al. 2012).

One possibility for telomere dynamics is a purely diffusive process, in which telomeres diffuse along the nuclear membrane and "stick" together when they meet other telomeres. However, it is also possible that telomeres are actively moved along the nuclear membrane. Proteins that link chromosomes to cytoskeletal elements have been discovered in many organisms (Scherthan 2007; Hiraoka and Dernburg 2009). These proteins, often containing SUN/KASH domains, create a bridge from chromosomes within the nucleus, through the nuclear membrane, to motor proteins outside of the nucleus. As these motor proteins walk along elements of the cytoskeleton, they drag the telomeres along the surface of the nucleus. If the underlying cytoskeleton is ordered and points in a consistent direction, directed motion results. However, if the cytoskeleton is completely disordered, on long enough length and timescales, an effective diffusion process is generated, with an effective diffusion constant that is potentially far larger than that generated by standard Brownian motion.

Mathematical modeling has again been vital in quantitatively assessing various different telomere-movement models, testing their compatibility with the available data, and making further predictions. Simulations of a minimal pure diffusive model (potentially facilitated by 
random motor-driven motion along the cytoskeleton) suggested that this would lead to large variations in the total time for bouquet formation, essentially because of the random nature of diffusive motion. Such large variations have not been observed experimentally (Fig. 3A) (Carlton et al. 2003) and hence such a model is not favored.

To better determine the relative parts played by diffusion and directed motion, it is important to understand the initial telomere configuration, that is, the arrangement of telomeres after attachment to the nuclear membrane but before bouquet formation has started. In many organisms, telomeres are not initially distributed uniformly over the nuclear surface but rather are preferentially found in a hemisphere (Cowan et al. 2001). This may be related to the Rabl configuration that is persistent in some organisms during interphase, in which the centromeres and telomeres occupy opposite sides of the nucleus. Furthermore, in the wheat-rye system that was studied by Richards et al. (2012), and potentially in many other organisms, it was found that this initial hemisphere does not have a preferred orientation with respect to the final bouquet site. This is a crucial difference to previous work in which it was found that if telomeres always occupy the same initial hemisphere, pure directed motion (without diffusion) cannot explain bouquet formation because the variation in bouquet formation time has become too small (Fig. 3B) (Carlton et al. 2003). For this reason, it was previously proposed, based on simulations, that it is a combination of directed motion and diffusion that explains bouquet formation (Carlton et al. 2003).

However, once it was appreciated that telomeres initially occupy random positions within a randomly oriented hemisphere on the nuclear membrane, it was possible (Richards et al. 2012) to perform more realistic, although still extremely minimal, spatiotemporal computer simulations of bouquet formation. These simulations included both directed motion and diffusion and
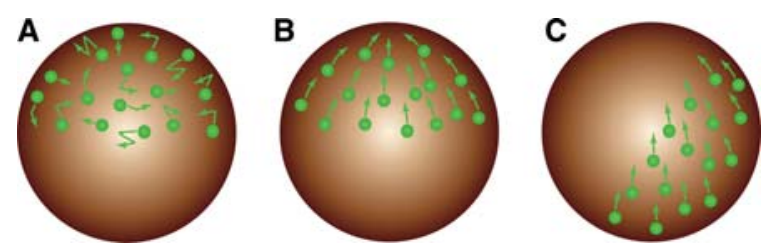

Figure 3. Possible models of how telomeres move along the nuclear membrane to form the bouquet. (A) Pure diffusion model with telomeres (green circles) randomly diffusing (jagged green arrows) on the nuclear membrane until they find the bouquet site. This model cannot explain bouquet formation because the variation in total time for bouquet formation is too large. This is true for any initial arrangement of telomeres. $(B)$ Directed motion (straight arrows) model, but with the telomeres always starting in the same hemisphere, cannot account for bouquet formation because now the variation in bouquet formation time is too small. $(C)$ The model preferred (Richards et al 2012) involves telomeres undergoing directed motion (straight arrows) that start from a randomly orientated hemisphere. Experimental data in wheat-rye directly support such a randomly oriented initial configuration. were compared to experimental measurements of telomere dynamics taken from wheat-rye. This approach allowed a reexamination of the relative effects of diffusion and directed motion. Interestingly, such an analysis predicted that a model without any diffusion at all, in which telomeres simply move directly toward the bouquet site, could explain much of the data, including both the total time for bouquet formation and the variation in this total time (Fig. 3C). The variation in the time for bouquet formation now originated from the random orientation of the initial hemisphere with respect to the bouquet formation site rather than from diffusion.

Although a directed motion model is useful for understanding the underlying mechanism of bouquet formation, it is perfectly possible that telomeres in reality do not move directly toward the bouquet site. The cytoskeletal element responsible for telomere motion (be it microtubules, actin, or something else) may well point on average toward the bouquet site but could have fluctuations in this direction. By incorporating this variation into the mathematical model, it was possible to predict the size of the variations. The best fit to the experimental data was found when the directionality of the cytoskeleton had a standard deviation of $\sim 40^{\circ}$. This model is similar to a case with directed motion toward the bouquet site plus effective diffusion but now only in a transverse direction (rather than the uniform diffusion considered previously). Crucially, this result shows that bouquet formation can proceed even with a relatively large variation in the direction of motion (Richards et al. 2012), emphasizing the robustness of bouquet formation. This conclusion underlines the importance of modeling in assessing the robustness of particular mechanisms to the presence of fluctuations.

\section{CONCLUSIONS}

In this chapter, we introduce and discuss two examples in which minimal mathematical modeling has helped to accelerate our understanding of the underlying biology. We now place these efforts in a broader context, discussing the current and potential future role of mathematical modeling in biology.

The modeling examples discussed previously were tightly focused, dealing with the epigenetic regulation of a particular developmental gene in Arabidopsis and the spatiotemporal dynamics of telomere bouquet formation in wheat-rye. Both are complex biological systems whose mode of action is far from clear and are therefore ideal candidates for mechanistic modeling. Indeed, many biological systems, even those with relatively few components, can give rise to unexpected behavior, that is not straightforward to understand intuitively. Modeling then comes into its own in rigorously showing whether a particular network of interactions can give rise to the observed behavior. Modeling of this sort can then generate predictions for experimental testing and subsequent model refinement. Such focused modeling can be contrasted with other computational approaches, such 
as those involving bioinformatics. We define bioinformatics here as the application of information sciences to the analysis of biological data, often involving the large-scale data generated in genomics and proteomics. Bioinformatics has, of course, been a highly successful methodology and clearly it overlaps to some extent with mathematical modeling. However, bioinformatics is often useful for discovering the components and interactions within a particular network, rather than for the rigorous dissection of how (or whether) the network can generate a particular behavior. It is probably fair to say that most experimental biologists are more comfortable dealing with the more traditional bioinformatics methods (such as sequence alignment) than with the less familiar tools of mathematical modeling (such as differential equations). Modeling approaches can, however, inject entirely new ways of thinking into biology, thereby accelerating the discovery of fundamental mechanisms. We believe that the two examples presented in this chapter provide modest examples of this process. In our opinion, it is the modeling approaches, currently under-represented in biology compared to bioinformatics, that will increasingly come to the fore during the next decade.

In addition to the difficulties faced by biologists in adapting to the methodologies used by modelers, mathematical modelers face equally significant challenges in approaching biological problems. Regardless of their exact origin, modelers (whether they are mathematicians, physicists, engineers, or computer scientists) are typically trained on clean, well-formulated problems. Moreover, most of these problems, although often hard to solve, were at least easy to state. Biology, of course, tends to defy such precise formulations and is full of problems that are frequently messy and often difficult to define clearly. This makes much of the problem in biological modeling actually one of setting up the problem in an appropriate way. Once this is done, making progress is often relatively simple. In principle, evolution should offer some insight into model formulation. As has been elegantly stated, "nothing in biology makes sense except in the light of evolution." Although undeniably true, whether such a perspective is practically useful in a modeling context is open to debate because it is often extremely difficult to distinguish between two potential mechanisms based purely on evolutionary arguments. Furthermore, whereas in physical science Occam's razor is a vital tool allowing unnecessarily complex theories to be dismissed without too much consideration, in biology its utility is much diminished, owing to the complexity of the systems and their evolutionary origin (Crick 1988).

At present, we must concede that the achievements of the modeling field have often been rather limited and not as impressive as some of its adherents would have us believe. Cases in which modeling has led to genuine, unexpected advances are still regrettably few in number, although this is perhaps not entirely surprising given that the modern era of modeling is relatively young. Nevertheless, the use of modeling methodologies would seem to offer one of the few compelling approaches to tame the immense complexity of biology. By adopting a systematic modular approach, we can hope to build up our fundamental understanding, with most progress being made on individual small-scale systems. Minimal, smallscale mathematical models can be validated by focused experiments and then periodically merged into largescale, comprehensive models able to probe the interactions among many systems. In this way, by building out from small-scale models, the difficulty of experimentally constraining the inevitably large number of parameters of large-scale models can at least be partly overcome. Despite recent pioneering efforts (Karr et al. 2012), comprehensive modeling of even the simplest bacterial cells is probably years away from substantial completion. In the short term, we believe that focused, small-scale efforts at fusing modeling and experimental biology, such as those detailed here, offer the best means to accelerate our understanding of complex biology. Simply put, understanding biology is a daunting task and it would seem wise to use all available tools to make the job a little easier.

\section{ACKNOWLEDGMENTS}

We thank Robert Ietswaart for a critical reading of the manuscript. This work was supported by BB/J004588/1 from the BBSRC, the John Innes Foundation, and the European Research Council.

\section{REFERENCES}

Angel A, Song J, Dean C, Howard M. 2011. A Polycomb-based switch underlying quantitative epigenetic memory. Nature 476: $105-108$.

Bastow R, Mylne JS, Lister C, Lippman Z, Martienssen RA, Dean C. 2004. Vernalization requires epigenetic silencing of FLC by histone methylation. Nature 427: 164-167.

Carlton PM, Cowan CR, Cande WZ. 2003. Directed motion of telomeres in the formation of the meiotic bouquet revealed by time course and simulation analysis. Mol Biol Cell 14: 2832-2843.

Cowan C, Carlton PM, Cande WZ. 2001. The polar arrangement of telomeres in interphase and meiosis. Rabl organization and the bouquet. Plant Physiol 125: 532-538.

Crevillen P, Dean C. 2010. Regulation of the floral repressor gene $F L C$ : The complexity of transcription in a chromatin context. Curr Opin Plant Biol 14: 38-44.

Crick F. 1988. What mad pursuit: A personal view of scientific discovery. Basic Books, New York.

De Lucia F, Crevillen P, Jones AME, Greb T, Dean C. 2008. A PHD-Polycomb repressive complex 2 triggers the epigenetic silencing of FLC during vernalization. Proc Natl Acad Sci 105: $16831-16836$.

Dodd IB, Micheelsen MA, Sneppen K, Thon G. 2007. Theoretical analysis of epigenetic cell memory by nucleosome modification. Cell 129: 813-822.

Greer E, Martin AC, Pendle A, Colas I, Jones AME, Moore G, Shaw P. 2012. The Ph1 locus suppresses Cdk2-type activity during premeiosis and meiosis in wheat. Plant Cell 24: 152162.

Hansen KH, Bracken AP, Pasini D, Dietrich N, Gehani SS, Monrad A, Rappsilber J, Lerdrup M, Helin K. 2008. A model for transmission of the H3K27me3 epigenetic mark. Nat Cell Biol 10: 1291-1300.

Harper L, Golubovskaya I, Cande WZ. 2004. A bouquet of chromosomes. J Cell Biol 117: 4025-4032. 
Hennig L, Derkacheva M. 2009. Diversity of Polycomb group complexes in plants: Same rules, different players? Trends Genet 25: 414-423.

Hiraoka Y, Dernburg AF. 2009. The SUN rises on meiotic chromosome dynamics. Dev Cell 17: 598-605.

Karr JR, Sanghvi JC, Macklin DN, Gutschow MV, Jacobs JM, Bolival B Jr, Assad-Garcia N, Glass JI, Covert MW. 2012. A whole-cell computational model predicts phenotype from genotype. Cell 150: 389-401.

Kaufman PD, Rando OJ. 2010. Chromatin as a potential carrier of heritable information. Curr Opin Cell Biol 22: 284 290.

Margueron R, Justin N, Ohno K, Sharpe ML, Son J, Drury WJI, Voigt P, Martin SR, Taylor WR, De Marco V, et al. 2009. Role of the Polycomb protein EED in the propagation of repressive histone marks. Nature 461: 762-767.

Moazed D. 2011. Mechanisms for the inheritance of chromatin states. Cell 146: 510-518.

Moore G, Shaw P. 2009. Improving the chances of finding the right partner. Curr Opin Genet Dev 19: 99-104.
Petruk S, Sedkov Y, Johnston DM, Hodgson JW, Black KL, Kovermann SK, Beck S, Canaani E, Brock HW, Mazo A. 2012. TrxG and PcG proteins but not methylated histones remain associated with DNA through replication. Cell 150: 922-933.

Ptashne M, Gann A. 2002. Genes \& signals. Cold Spring Harbor Laboratory Press, Cold Spring Harbor, New York.

Richards DM, Greer E, Martin AC, Moore G, Shaw PJ, Howard M. 2012. Quantitative dynamics of telomere bouquet formation. PLoS Comput Biol 8: e1002812.

Scherthan H. 2001. A bouquet makes ends meet. Nat Rev Mol Cell Biol 2: 621-627.

Scherthan H. 2007. Telomere attachment and clustering during meiosis. Cell Mol Life Sci 64: 117-124.

Schwartz YB, Pirrotta V. 2007. Polycomb silencing mechanisms and the management of genomic programmes. Nat Rev Genet 8: $9-22$.

Song J, Angel A, Howard M, Dean C. 2012. Vernalization: A cold-induced epigenetic switch. J Cell Sci 125: 3723-3731.

Zickler D, Kleckner N. 1998. The leptotene-zygotene transition of meiosis. Аnпu Rev Genet 32: 619-697. 


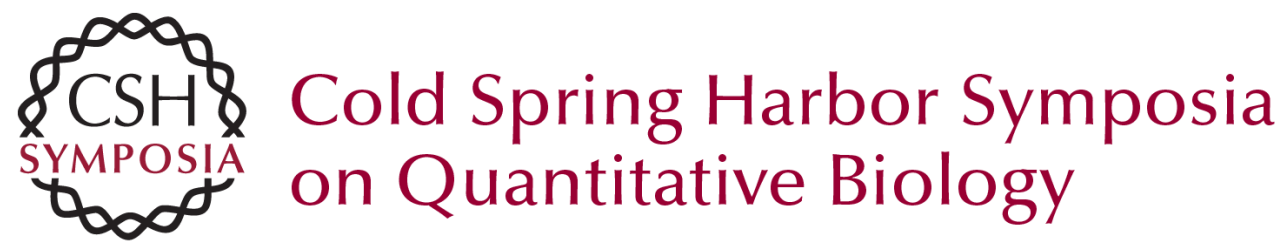

\section{Illustrations of Mathematical Modeling in Biology: Epigenetics, Meiosis, and an Outlook}

D. Richards, S. Berry and M. Howard

Cold Spring Harb Symp Quant Biol 2012 77: 175-181 originally published online January 22, 2013

Access the most recent version at doi:10.1101/sqb.2013.77.015941

References This article cites 24 articles, 5 of which can be accessed free at: http://symposium.cshlp.org/content/77/175.full.html\#ref-list-1

\section{License}

Email Alerting Receive free email alerts when new articles cite this article - sign up in Service the box at the top right corner of the article or click here. 